\title{
Have dentists a role in identifying mentally ill patients?
}

\author{
A preliminary communication on whether general dental practitioners have a role in identifying dental patients with \\ mental health problems by F. Lloyd-Williams, C. Dowrick, D. Hillon, G. Humphris, G. Moulding and R. Ireland \\ Br Dent J 2001; 191: 625-629
}

\author{
Objective \\ To examine whether general dental practitioners regard \\ themselves as having a role in identifying dental patients with \\ mental health problems.
}

\section{Method}

Telephone interviews were conducted with, and postal questionnaires were sent to, a random sample $(n=94)$ of general dental practitioners on Health Authority lists within the Mersey Region. A total of 84 general dental practitioners responded, resulting in an $89 \%$ response rate.

\section{Results}

The majority of general dental practitioners had encountered patients with mental health problems ( $78 \%$ of interviewees, $56 \%$ of questionnaire responders). However, nearly half of the interviewees $(46 \%)$ do not refer patients with mental health problems. When referrals to dental specialists were made, they tended to be regarding a physical manifestation rather than a psychological one. The majority of interviewees and questionnaire responders (55\% and $82 \%$ respectively) expressed a 'positive' response to the development of a referral role. Nevertheless, there were some reservations concerning the practicalities of its development and implementation.

\section{Conclusion}

The majority of general dental practitioners consider a role in identifying patients with possible mental health problems. This role might include, being able to identify patients with undiagnosed mental health problems, and being aware of dental conditions which may be caused by mental health problems. To achieve this, inter-professional co-operation between general dental practitioners and other health professionals requires development.

\section{Comment}

$\mathrm{n}$ recent times there has been a shift in the perception of the role of the dentist in
percention general practice. The dentist is no longer perceived as an individual working on his own filling teeth, but rather a health professional working in a primary care team, interacting with colleagues and providing complete patient care. Recognising these developments within general dental practice allowed Lloyd-Williams et al. to pose the question: 'Do general dental practitioners have a role in identifying dental patients with mental health problems?'

In a survey of dentists in general practice the authors set about to discover whether dentists were aware of patients' mental health problems, how they identified patients and if they felt they had a part to play. The results suggest that dentists did encounter patients with mental health problems ranging from dental anxiety to depression to psychosomatic disorders. The dentists seemed to have little difficulty in identifying patients with mental health difficulties but remained hesitant in broaching the subject with their patients. In general the dentists felt ill-prepared and inadequately trained. Consequently, dentists recommended that patients attended their general medical practitioner or referred patients with psychosomatic problems (eg burning mouth syndrome, phantom toothache) to dental specialists while others attempted to help patients by using physical treatments for psychological problems.

Dentists in general practice do encounter patients with psychological problems. These may include those with anxiety, prolonged bereavement reactions or patients whose degree of self-reproachfulness suggests a severe clinical depression. Dentists may also be the first to encounter an adolescent or young adult who is preoccupied and obsessed by the appearance of his teeth (dysmorphophobia). The delusional quality of the belief that the teeth are deformed in some way may suggest that the dysmorphophobia is a prodromal symptom of schizophrenia. The need for dental practitioners to identify these patients and to refer them for appropriate care is essential. The authors propose the need for additional communication skills' training and the introduction of specific referral guidelines. They recommend that referral networks between those in general dental practice and those in general medical practice are developed, strengthened and maintained. These are important recommendations if dentists in general dental practice are to play a role in identifying and caring of patients with mental health problems.

\section{Ruth Freeman}

Professor of Dental Public Health, School of Dentistry, The Queen's University of Belfast 